\title{
Erratum to: Galactic Radio Astronomy
}

Yoshiaki Sofue

Erratum to:

Y. Sofue, Galactic Radio Astronomy, Lecture Notes in Physics 935, https://doi.org/10.1007/978-981-10-3445-9

The original version of this book was published without printing chapter abstract. The abstracts for each chapter are updated in the front matter in the current version of the book.

The updated online version of the book can be found at https://doi.org/10.1007/978-981-10-3445-9 